MATEC Web of Conferences 32, 02005 (2015)

DOI: $10.1051 /$ matecconf $/ 20153202005$

(O) Owned by the authors, published by EDP Sciences, 2015

\title{
Femtosecond Laser Induced Underwater Superoleophobic Surfaces
}

\author{
Jiale Yong ${ }^{1}$, Feng Chen ${ }^{1, a}$, Qing Yang ${ }^{1}$ \\ ${ }^{1}$ State Key Laboratory for Manufacturing System Engineering \& Key Laboratory of Photonics Technology for Information of Shaanxi \\ Province, School of Electronics \& Information Engineering, Xi'an Jiaotong University, Xi'an, 710049, P. R. China
}

\begin{abstract}
Femtosecond laser microfabrication has been recently utilized in interface science to modify the liquid wettability of solid surfaces. Silicon surface with hierarchical micro/nanostructure is fabricated by a femtosecond laser. Similar to the fish's scales, the laser-induced surface shows superhydrophilicity in air and superoleophobicity underwater. The oil contact angles can reach up to $159.4 \pm 1^{\circ}$ for the 1,2-dichloroethane droplets in water. Besides, the surface exhibits ultralow oil-adhesion. In the oil/water/solid three-phase system, water can be trapped in the hierarchical rough structure and forms a repulsive oil layer according to underwater Cassie's theory. The contact area between the asprepared surface and oil droplet is significantly reduced, resulting in superoleophobicity and ultralow oil-adhesion in water. In addition, transparent underwater superoleophobic and anti-oil surfaces are achieved on silica glass surfaces by femtosecond laser ablation. This transparent property is attributed to the presence of the water environment because scattering and refraction are effectively weakened. The presented method is simple and can accurately control the processing location, which may have widely potential applications in, for instance, microfluidics, biotechnologies, and antifouling coatings.
\end{abstract}

\section{References}

1. A. Mecke, I. Lee, J.R. Baker jr., M.M. Banaszak Holl, B.G. Orr, Eur. Phys. J. E 14, 7 (2004) J. Yong, F. Chen, Q. Yang, D. Zhang, U. Farooq, G. Du, X. Hou, J. Mater. Chem. A 2, 8790 (2014)

2. J. Yong, F. Chen, Q. Yang, G. Du, C. Shan, H. Bian, U. Farooq, X. Hou, J. Mater. Chem. A 3, 9379 (2015)

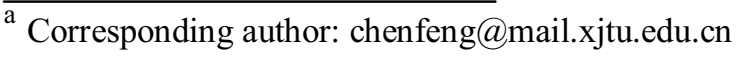

This is an Open Access article distributed under the terms of the Creative Commons Attribution License 4.0, which permits unrestricted use, distribution, and reproduction in any medium, provided the original work is properly cited. 